\title{
Prevalencia de Enterococos resistentes a antibióticos en aguas servidas en el norte de Chile
}

\author{
Juan Silva $A^{a}$, Patricio Loyola $S^{b}$, Juan $G$ alleguillos $\mathbf{O}^{\text {b }}$, \\ Yara Rodríguez G C, Patricia Colque-N avarro ${ }^{1, d}$, \\ Roland Möllby ${ }^{1, e}$, Inger Kühn ${ }^{1, f}$. \\ Prevalence of antibiotic resistant \\ Enterococcus spp in waste waters \\ in the north of Chile
}

Background: There is little information available in Chile on the distribution of Enterococcus spp in waste water and its implications in transmission of antibiotic resistance through the water cycle. Enterococcus spp are common in nosocomial infections and may spread antibiotic resistance through the food chain. Aim: To determine the presence of antibiotic resistant Enterococcus spp in the sewage of Antofagasta, Chile. Material and Methods: Samples of sewage from two sewage treatment plants and from the Public Hospital of Antofagasta collector were obtained. Enterococcus spp were isolated on $\mathrm{m}$-Enterococcus agar containing ampicillin, vancomycin and streptomycin. The isolates were identified and subjected to biochemical typing (PhPlate). Minimal inhibitory concentration determination was performed by agar dilution technique. Results: High counts of resistant Enterococcus spp were found on the streptomycin plates, lower on ampicillin and very low on vancomycin plates. A total of 63 Enterococcus spp strains were typed and the identification showed 5 different species; E faecalis (65\%), E faecium (14\%), E hirae (13\%), E durans (6\%) and E gallinarum (2\%). The typing revealed a high diversity among the isolates. Two biochemical phenotypes were predominant, C1 (21 strains) and C6 (7 strains). Both were highly resistant to gentamycin and streptomycin; moderately resistant to ampicillin, cloramphenicol, tetracycline and ciprofloxacin, and with intermediate susceptibility to vancomycin. Both phenotypes were found in the sewage of the hospital collector and in the treatment plants. Conclusions: In the sewage of Antofagasta we found dominating phenotypes of multiresistant Enterococcus spp. Sewage could be an important way of transmission of these microorganisms (Rev Méd Chile 2005; 133: 1201-10).

(Key Words: Enterococcus; Enterococcus faecalis; Enterococcus faecium; Sewage; Waste products)

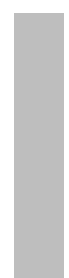

Recibido el 30 de diciembre, 2004. Aceptado el 6 de junio, 2005.

Financiado por Proyecto PROIM N ${ }^{\circ} 1357$, Dirección de Investigación de la Universidad de Antofagasta y Proyecto \# C 13955, Fundación Andes.

Departamento de Tecnología Médica - INDES, Universidad de Antofagasta, Chile. ${ }^{1}$ Microbiology and Tumorbiology Center, Karolinska Institute, Estocolmo, Suecia.

aTecnólogo Médico, Magíster en Microbiología, PhD en Biología. b Tecnólogo Médico. ${ }^{\mathrm{C} B i o ́ l o g a . ~}$ dIngeniero Agrónomo, Licenciado en Microbiología. eMédico, PhD en Biología. ${ }^{\mathrm{P} h D}$ en Microbiología.

Correspondencia a: Dr. Juan Silva A. Departamento de Tecnología Médica-INDES, Universidad de Antofagasta. Avenida Coloso s/n Antofagasta, Chile. Fonofax: (56-55) 637207. E-mail: jsilva@uantof.cl 
L os enterococos forman parte de la flora normal del intestino del hombre y los animales, por lo que son liberados directamente al medio ambiente o a través de emisarios de aguas servidas, donde pueden sobrevivir por largos periodos de tiempo ${ }^{1}$. A pesar de su escasa virulencia, cepas de enterococos resistentes a antibióticos han emergido como patógenos importantes en infecciones nosocomiales y de la comunidad, en el últimos años ${ }^{2-5}$. La evolución y diseminación de la resistencia ha sido favorecida por el uso de antibióticos en el medio hospitalario, como así también en la crianza de animales ${ }^{6-8}$. Además, estos microorganismos tienen una gran capacidad de adquirir y transferir resistencia a las drogas antimicrobianas a elevadas concentraciones, lo que dificulta su tratamiento ${ }^{9-}$ 11. Durante la última década, la investigación mundial ha sido enfocada al estudio de los enterococos resistentes a vancomicina (EVR), debido a la aparición y diseminación de EVR en los hospitales, que constituyen un gran riesgo para los pacientes hospitalizados ${ }^{12}$. Recientes informes del Center of Disease Control (CDC) de Estados Unidos, señalan que $25 \%$ de las infecciones por EVR ocurren en pacientes de los Servicios de Cuidados Intensivos ${ }^{13}$. Hasta hace poco tiempo, la vancomicina era considerada el último recurso terapéutico para tratar infecciones por enterococos y estafilococos meticilino resistente.

Algunos autores han señalado que una posible ruta de transmisión de los determinantes genéticos de resistencia a vancomicina, ha ocurrido desde los animales a los humanos, vía cadena alimentaria ${ }^{14}$. En algunos países europeos, el amplio y continuo uso de antibióticos, como promotores del crecimiento de animales de crianza, ha contribuido a la selección de cepas resistentes a las drogas antimicrobianas usadas en la terapia humana, y tales cepas bacterianas son encontradas en animales y en humanos ${ }^{15,16}$. En cambio, en los Estados Unidos la diseminación de cepas de EVR en los hospitales ha sido atribuida al uso excesivo de vancomicina y antibióticos de amplio espectro, como las cefalosporinas usadas en el medio hospitalario y que posteriormente, las cepas de EVR son diseminadas al medio ambiente, a través de las aguas servidas de los hospitales ${ }^{17,18}$. Recientemente, Rice y col ${ }^{13}$ han demostrado que el uso de ciertos antibióticos ß-lactámicos en ratones, como ceftriaxona y cefo- tetan, contribuyen a un alto nivel de colonización gastrointestinal por cepas de EVR.

Diversos estudios han demostrado que las aguas servidas constituyen un reservorio importante de cepas de enterococos resistentes a los antibióticos, siendo $\mathrm{E}$ faecalis y $\mathrm{E}$ faecium las especies aisladas con mayor frecuencia ${ }^{19-22}$. También, se ha señalado una posible ruta de transmisión de enterococos resistentes, desde pacientes hospitalizados a los emisarios de aguas servidas del hospital y aguas servidas urbanas, más tarde a través de la vía de las plantas de tratamiento para aguas superficiales pueden regresar al ser humano ${ }^{18}$.

En Chile, existe escasa información sobre la estructura poblacional de las cepas de enterococos, así como también de su tasa de resistencia a los antibióticos. Tampoco existen estudios sobre la vía de transmisión de estos microorganismos. Es necesaria una continua vigilancia para conocer si la transmisión ocurre vía cadena alimentaria o se generan en el medio ambiente. En hospitales del norte de Chile, hemos aislado algunas cepas de enterococos con resistencia a aminoglicósidos a elevadas concentraciones, en menor proporción a ampicilina y un escaso número de cepas con resistencia intermedia a vancomicina ${ }^{23}$.

El objetivo de este trabajo fue estudiar: i) la prevalencia de poblaciones de enterococos resistentes a los antibióticos en aguas servidas de Antofagasta, ii) la distribución de sus especies y resistencia a los antibióticos, y iii) la diversidad y similitud de los fenotipos prevalentes.

\section{MATERIAL Y MÉTODO}

Recolección de las muestras. Se recolectaron muestras de aguas servidas del colector del Hospital de Antofagasta y de las plantas de tratamiento Cascal Norte y Cascal Sur, en dos períodos distintos, con el objeto de recabar una información preliminar. Las muestras de aguas servidas sin tratar, fueron recolectadas, por duplicados, en frascos estériles de 500 $\mathrm{ml}$, con las medidas de bioseguridad necesarias y fueron transportadas mediante bolsas plásticas en cajas refrigeradas. Las muestras fueron procesadas dentro de 2 horas después de su obtención, en el Laboratorio de Microbiología Ambiental - INDES, Universidad de Antofagasta. 
Aislamientos de cepas de enterococos resistentes. Las muestras fueron homogeneizadas a temperatura ambiente y diluidas en $180 \mathrm{ml}$ de suero fisiológico estéril contenidos en botellas de vidrio de $300 \mathrm{ml}$ (diluciones $10^{-1}$ a $10^{-6}$ ); $50 \mathrm{ml}$ de cada una de las diluciones fueron filtradas por medio de filtros de membrana de 0,45 (Millipore) al vacío. Cada una de las membranas fueron colocadas en agar Tripticasa e incubadas a $37^{\circ} \mathrm{C}$ durante 2 horas. Las membranas fueron retiradas y colocadas en placas de agar selectivo m-Enterococus (Merck) con y sin antibióticos, incubándolas a $37^{\circ} \mathrm{C}$ durante $48 \mathrm{~h}$. Finalmente, las membranas fueron colocadas en placas de agar bilis esculina $\mathrm{e}$ incubadas a $44^{\circ} \mathrm{C}$ durante $2 \mathrm{~h}$. Las placas con antibiótico contenían ampicilina $25 \mu \mathrm{g} / \mathrm{ml}$, vancomicina $10 \mu \mathrm{g} / \mathrm{ml}$ y estreptomicina $25 \mu \mathrm{g} / \mathrm{ml}$, respectivamente.

Identificación de los fenotipos bioquímicos de Enterococci. La determinación de los fenotipos bioquímicos (biochemical fingerprinting) se hizo por el método de Ph-RF (Phene Plate Techniques AB, Suecia), de acuerdo a recomendaciones de Kühn y $\mathrm{Col}^{24}$. A partir de las placas con agar bilis esculina, un total de 8 a 9 colonias negras azabache reconocidas presuntivamente como enterococos, fueron seleccionadas y tipificadas por el sistema $\mathrm{Ph}-\mathrm{RF}$. Las colonias fueron inoculadas en microplacas, que contienen 96 pocillos con 11 sustratos deshidratados seleccionados, los cuales proveen un alto nivel de discriminación para las especies de enterococos. Las microplacas del Ph-Plate fueron inoculadas, a partir de una colonia aislada repicada desde la placa de cultivo y se suspendió en el primer pocillo de cada columna de la placa, después se inocularon los demás pocillos. Las microplacas se incubaron a $37^{\circ} \mathrm{C}$ hasta un total de $64 \mathrm{~h}$. La cinética de las reacciones fue evaluada midiendo los valores de absorbancia a las 16, $40 \mathrm{y}$ $64 \mathrm{~h}$. Un fingerprinting bioquímico (fenotipo) se calculó como el valor promedio para cada reactivo de las 3 lecturas, mediante el programa PhP-WIM4. La relación de los diferentes fenotipos observados fue ilustrada en un dendograma (Figura 1).

Caracterización de las especies de enterococos. Las cepas de fenotipos seleccionados por el sistema $\mathrm{Ph}-\mathrm{RF}$, se identificaron mediante pruebas bioquímicas, como crecimiento en $\mathrm{NaCl} 6,5 \%$ y telurito, hidrólisis de diversos carbohidratos y aminoácidos y otras pruebas bioquímicas ${ }^{25}$. También, el $\mathrm{Ph}$ Plate permitió la identificación primaria, al comparar los fenotipos de cepas desconocidas de enterococos con aquellas de un set de referencia de cepas conocidas, que han sido previamente caracterizadas por el programa PhP-WIN.

Determinación de la resistencia a los antibióticos. A las cepas de enterococos identificadas se les determinó la concentración mínima inhibitoria (CMI) a varios antibióticos, de acuerdo a lo descrito en trabajos anteriores ${ }^{26}$, mediante técnica de dilución en agar y siguiendo las recomendaciones de la NCCSL (2002) ${ }^{27}$. Se emplearon los siguientes agentes antimicrobianos: ampicilina (AM), vancomicina (V), gentamicina (GM), estreptomicina (ES), cloranfenicol (C), ciprofloxacino $(\mathrm{CIP})$, penicilina $(\mathrm{P})$, tetraciclina $(\mathrm{T})$ y eritromicina (ER). Se establecieron los porcentajes y patrones de cepas resistentes a estos antibióticos. También, a las cepas se les determinó la resistencia a aminoglicósidos a altos niveles de concentración. Los resultados de la resistencia fueron descritos como la $\mathrm{CMI}_{50} \mathrm{Y} \mathrm{CM}_{90}$ (Tabla 3). En todos los ensayos se incluyó la cepa de E faecalis ATCC 29212 como control.

\section{RESULTADOS}

Los resultados obtenidos en nuestro estudio nos indican la presencia de cepas de enterococos resistentes a antibióticos en la mayoría de las muestras de aguas servidas analizadas (Tabla 1). Los promedios de los recuentos bacterianos fueron muy similares en las muestras obtenidas de las plantas tratamientos y en el colector del hospital, fluctuando entre 5,1 a 6,2 x $10^{6} \mathrm{UFC} / \mathrm{ml}$. Los mayores recuentos de enterococos resistentes se apreciaron en las placas de estreptomicina (3,8 a $4,4 \times 10^{5} \mathrm{UFC} / \mathrm{ml}$ ) y menor proporción, en las placas de ampicilina (1,8 a 2,5 x $\left.10^{3} \mathrm{UFC} / \mathrm{ml}\right)$. En el caso de vancomicina, los recuentos de enterococos resistentes fueron muy bajos (3 a 6 UFC x $100 \mathrm{ml}$ ).

Entre las cepas resistentes, se identificaron 5 diferentes especies del género Enterococus, las que se presentan en la Tabla 2. La especie más frecuentemente aislada fue E faecalis (65\%), y en menor proporción $\mathrm{E}$ faecium (14\%), E hirae 


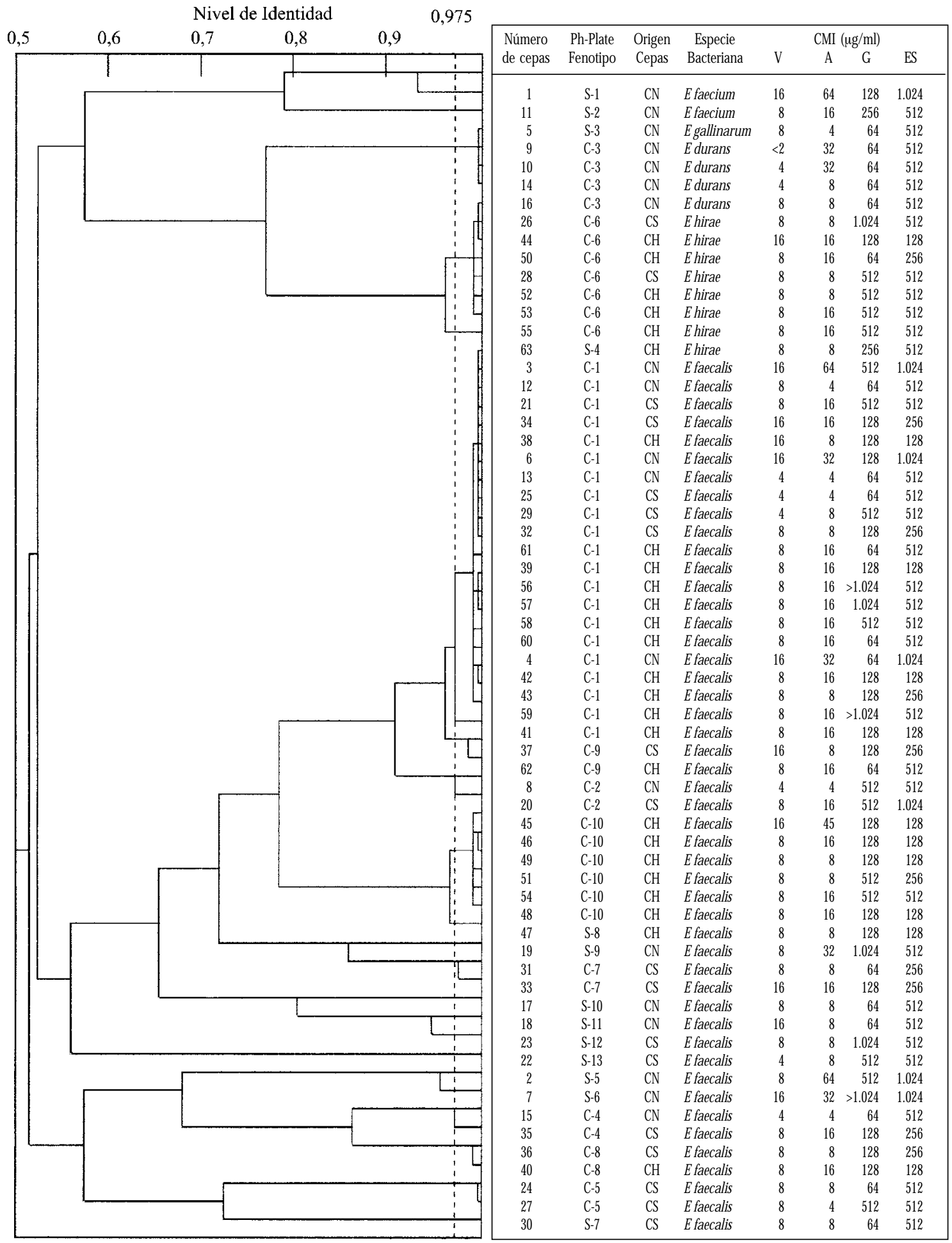

Figura 1. Dendograma de cepas de Enterococcus resistentes a los antibicóticos en aguas servidas de plantas de tratamientos y colector del Hospital Regional.

$\mathrm{C}=\mathrm{PhP}$ Fenotipos. S= Cepas no relacionadas. $\mathrm{CH}=$ Colector del Hospital. $\mathrm{CN}=$ Planta Tratamiento «Cascal Norte». CS= Planta Tratamiento «Cascal Sur» V= vancomicina. A= ampicilina. $\mathrm{G}=$ gentamicina. $\mathrm{ES}=$ estreptomicina. 
(13\%), E durans (6\%) y E gallinarum (2\%). Sin embargo, en las aguas servidas del colector del hospital, la segunda especie más frecuente correspondió a E hirae (23\%). También, cabe destacar que E durans (21\%) y E gallinarun (5\%) fueron detectadas solamente en aguas servidas de la planta de tratamiento Cascal Norte.
En los ensayos de susceptibilidad a los antibióticos, con las 63 cepas de enterococos frente a las diferentes drogas antimicrobianas, se observó una elevada tasa de resistencia de alto nivel a los aminoglicósidos. En la Tabla 3, se muestra que las cepas de $\mathrm{E}$ faecalis presentaron en $68,3 \%$ resistencia a gentamicina y 100\% a estreptomicina. Las

Tabla 1. Valores promedios de los recuentos de cepas de Enterococcus spp en agar $m$-Enterococcus con y sin antibióticos, según sitio de muestreo

\begin{tabular}{|lccccc|}
\hline Origen & No de & \multicolumn{3}{c|}{ Recuento (UFC/100 ml) } \\
& muestras & A & V $^{*}$ & Es & Total \\
\hline Planta Cascal Norte & 2 & $2,5 \times 10^{3}$ & $6^{* *}$ & $4,4 \times 10^{5}$ & $6,0 \times 10^{6}$ \\
Colector Hospital Regional & 2 & $1,8 \times 10^{3}$ & $3^{* *}$ & $4,2 \times 10^{5}$ & $5,1 \times 10^{6}$ \\
Planta Cascal Sur & 2 & $2,1 \times 10^{3}$ & $3^{* *}$ & $3,8 \times 10^{5}$ & $6,2 \times 10^{6}$ \\
\hline
\end{tabular}

${ }^{*}$ Colonias en EV10. * Total de cepas aisladas.

$\mathrm{A}=$ ampicilina. $\mathrm{V}=$ vancomicina. $\mathrm{Es}=$ estreptomicina

Tabla 2. D istribución de las especies de Enterococcus aisladas de las aguas servidas, según su origen de obtención

\begin{tabular}{|lrrrrrrrr|}
\hline $\begin{array}{l}\text { Especies } \\
\text { Bacterianas }\end{array}$ & \multicolumn{2}{c}{$\begin{array}{c}\text { Cascal Norte } \\
\text { № }\end{array}$} & \multicolumn{2}{c}{$\begin{array}{c}\text { Cascal Sur } \\
\text { No }\end{array}$} & № & $\%$ & Colector Hospital & \multicolumn{2}{c|}{ Total } \\
№ & $\%$ & № & $\%$ \\
\hline E faecalis & 12 & 63 & 14 & 78 & 15 & 58 & 41 & 65 \\
E faecium & 2 & 11 & 2 & 11 & 5 & 19 & 9 & 14 \\
E hirae & 0 & 0 & 2 & 11 & 6 & 23 & 8 & 13 \\
E durans & 4 & 21 & 0 & 0 & 0 & 0 & 4 & 6 \\
E gallinarun & 1 & 5 & 0 & 0 & 0 & 0 & 1 & 2 \\
Total & 19 & 100 & 18 & 100 & 26 & 100 & 63 & 100 \\
\hline
\end{tabular}

Tabla 3. D istribución de las $\mathrm{CMI}_{50}$ y $\mathrm{CMI}_{90}$ de cepas de $\mathrm{E}$ faecalis y $\mathrm{E}$ faecium aisladas de aguas servidas de la ciudad de Antofagasta (2003)

\begin{tabular}{|lcrrrrrr|}
\hline \multirow{2}{*}{$\begin{array}{l}\text { Drogas } \\
\text { Antimicrobianas }\end{array}$} & \multicolumn{3}{c}{ Rango faecalis } & \multicolumn{3}{c|}{ E faecium } \\
& $\mathrm{CM}_{50}$ & $\mathrm{CM}_{90}$ & \% Resistencia & $\mathrm{CMI}_{50}$ & $\mathrm{CM}_{90}$ & \% Resistencia \\
\hline Vancomicina & $2-16$ & 8 & 16 & 0 & 8 & 8 & 0 \\
Ampicilina & $4-64$ & 16 & 32 & 17,0 & 16 & 32 & 44,0 \\
Penicilina & $2-128$ & 8 & 32 & 26,8 & 8 & 32 & 22,0 \\
Tetraciclina & $2-128$ & 16 & 32 & 36,6 & 16 & 32 & 33,3 \\
Ciprofloxacino & $2-128$ & 8 & 8 & 60,0 & 8 & 8 & 44,4 \\
Cloranfenicol & $2-256$ & 64 & 64 & 90,2 & 64 & 64 & 88,9 \\
Gentamicina & $64-1.024$ & 128 & 512 & 68,3 & 128 & 256 & 100,0 \\
Estreptomicina & $128-2.048$ & 512 & 1.024 & 87,8 & 512 & 512 & 100,0 \\
Eritromicina & $2-128$ & 4 & 8 & 50,5 & 8 & 8 & 66,7 \\
\hline
\end{tabular}


cepas de E faecium exhibieron $87,8 \%$ de resistencia a gentamicina y $100 \%$ a estreptomicina. También se apreciaron en las mismas especies, elevada resistencia a cloranfenicol, 90,2\% y 88,9\%, respectivamente. Además, destacó la ausencia de cepas resistentes a vancomicina, detectándose solamente algunas cepas con resistencia intermedia. Los valores de la $\mathrm{CMI}_{50}$ y $\mathrm{CMI}_{90}$ estuvieron muy cercanos entre sí, indicando una alta resistencia a los antibióticos aminoglicósidos, cloranfenicol y una resistencia moderada a ciprofloxacino, tetraciclina y ampicilina.

El sistema de Ph-Plate ha sido usado con éxito en estudios ecológicos y epidemiológicos de enterococos ${ }^{1,28}$. En la Tabla 4, se muestran los resultados de los ensayos de Ph-Plate, los que permitieron detectar 23 fenotipos diferentes, de los cuales 10 estaban representados con 2 o más cepas similares y 13 constituidas individuales ( $\sin$ relación con otra cepa). El fenotipo C1 fue el más frecuentemente aislado, con 21 cepas (distribuidos en las 3 fuentes de aguas servidas), seguido de C6 con 7 cepas y otros en menor proporción.

En la Figura 1, se presenta el dendograma de las cepas de enterococos estudiadas. En este dendograma se agrupan las cepas de enterococos en cuanto su grado de relación en diferentes fenotipos. Se puede apreciar que existen fenotipos prevalentes que se encuentran en las plantas de tratamientos, Cascal Norte y Cascal Sur, y en las aguas del colector del hospital y que, además, exhiben una elevada tasa de resistencia de alto nivel a estreptomicina y gentamicina a altas concentraciones.

Tabla 4. D istribución de fenotipos de Enterococcus resistentes según origen de las muestras de aguas servidas de la ciudad de Antofagasta

\begin{tabular}{|lcccc|}
\hline $\begin{array}{l}\text { Fenotipo } \\
\text { (Ph-Plate) }\end{array}$ & $\begin{array}{c}\text { Origen de las Cepas } \\
\text { Cascal } \\
\text { Norte }\end{array}$ & $\begin{array}{c}\text { Hospital } \\
\text { Regional }\end{array}$ & $\begin{array}{c}\text { Caspal } \\
\text { Sur }\end{array}$ & Total \\
\hline C1 & 5 & 11 & 5 & 21 \\
C2 & 1 & 0 & 1 & 2 \\
C3 & 4 & 0 & 0 & 4 \\
C4 & 1 & 0 & 1 & 2 \\
C5 & 0 & 0 & 2 & 2 \\
C6 & 0 & 5 & 2 & 7 \\
C7 & 0 & 0 & 2 & 2 \\
C8 & 0 & 1 & 1 & 2 \\
C9 & 0 & 1 & 1 & 2 \\
C10 & 0 & - & 0 & 6 \\
S1 & 1 & - & - & 1 \\
S2 & 1 & - & - & 1 \\
S3 & 1 & 1 & - & 1 \\
S4 & - & - & - & 1 \\
S5 & 1 & - & - & 1 \\
S6 & - & - & 1 \\
S7 & 1 & 1 & 1 & 1 \\
S8 & - & 1 & - & 1 \\
S9 & - & - & - & 1 \\
S10 & - & - & - & 1 \\
S11 & - & - & 1 & 1 \\
S12 & 1 & - & & \\
S13 & 1 & - & - & \\
& - & & 1 & \\
\hline
\end{tabular}




\section{DISCUSIÓN}

Se ha encontrado una alta prevalencia de cepas de enterococos resistentes a los antibióticos aminoglicósidos en las aguas servidas de la ciudad de Antofagasta y esta resistencia es mucho mayor a la observada en enterococos aislados de muestras clínicas en Chile $^{29}$. Es posible que su mayor aislamiento en este estudio, se deba al uso de un medio de enriquecimiento y su posterior cultivo en medio selectivo para enterococos adicionado con estreptomicina. En Suecia se han realizado estudios con la misma metodología, encontrándose una alta prevalencia de enterococos vancomicina resistentes ${ }^{1}$.

Los recuentos totales de enterococos en los medios selectivos, indicaron niveles de cepas resistentes que fluctuaron entre $10^{3}$ y $10^{5} \mathrm{UFC} / \mathrm{ml}$ en las aguas servidas, no observándose grandes diferencias en relación al origen de las muestras. Resultados similares han sido descrito por Blanch y $\mathrm{col}^{22}$. Los recuentos de cepas de enterococos resistentes a ampicilina fueron sobre $10^{3} \mathrm{UFC} / \mathrm{ml}$ y para estreptomicina de $10^{5} \mathrm{UFC} / \mathrm{ml}$. Esta diferencia en los recuentos se relacionan con la actividad que estos dos antibióticos tienen sobre cepas de enterococos de origen clínico a nivel nacional ${ }^{29}$.

La pruebas de identificación de las 63 cepas de enterococos seleccionadas, nos permitieron caracterizar 5 especies diferentes de enterococos: $\mathrm{E}$ faecalis, E faecium, E hirae, E durans y E gallinarun. En las muestras de aguas se detectó una elevada frecuencia de aislamiento de E faecalis, lo que concuerda con lo descrito por diversos autores $^{1,25}$ que coinciden además, con el aislamiento de E faecium como segunda frecuencia.

Con relación a la resistencia a los antibióticos, en este estudio se pudo apreciar ausencia de resistencia a vancomicina. Solamente se detectó $19 \%$ de cepas de enterococos con resistencia intermedia a vancomicina (CMI de $16 \mu \mathrm{g} / \mathrm{ml}$ ). Esto reviste gran interés, pues es posible que en un corto tiempo surgan cepas de enterococos que alcancen valores más altos de resistencia a vancomicina. Este resultado contrasta con los reportes de Suecia, en los que se ha encontrado una alta prevalencia de cepas de enterococos resistentes a vancomicina en aguas servidas ${ }^{1}$. La ausencia de cepas resistentes a vancomicina también ha sido mencionada en otros trabajos nacionales ${ }^{29}$. Por el contrario, Mella y $\operatorname{col}^{30}$ describieron el hallazgo de dos cepas de E faecium resistentes a vancomicina, en las que se detectó el gen vanB ${ }^{30}$. También, el Instituto de Salud Pública de Chile implementó un sistema de vigilancia para controlar la diseminación y disminuir el impacto de la resistencia a vancomicina en cepas de enterococos. En el año 2000, se encontró una prevalencia de 11\% de EVR y en 2001 de $15 \%$, de un total de 150 y 206 cepas recibidas, respectivamente, que si bien es cierto es una muestra sesgada, podría estar señalando un aumento paulatino del aislamiento de EVR en todos los hospitales de Chile ${ }^{31}$. Por lo tanto, es importante evaluar periódicamente la actividad de este antibiótico frente a estas especies bacterianas, y especialmente, aumentar la vigilancia ambiental $\mathrm{y}$ en la comunidad.

En cuanto a la resistencia a penicilina y ampicilina, se observó una resistencia moderada en las cepas de enterococos y, además, a bajas concentraciones $\left(\mathrm{CMI}_{90}=32 \mu \mathrm{g} / \mathrm{ml}\right)$. Frente a ampicilina, E faecium presentó una mayor resistencia con $44 \%$, en cambio, E faecalis sólo alcanzó a $17 \%$, lo que concuerda con los resultados descritos en otros estudios, que muestran que la mayor resistencia a los ß-lactámicos se presenta en $\mathrm{E}$ faecium $^{9}$. Es posible que la resistencia a ampicilina esté mediada por la presencia de una ß-lactamasa, como ha sido descrita por otros investigadores ${ }^{32,33}$. Otro punto importante para destacar, es la elevada resistencia de alto nivel que presentaron las cepas aisladas a los aminoglicósidos; gentamicina (128 a $1.024 \mu \mathrm{g} / \mathrm{ml}$ ) y estreptomicina (64 a $1.024 \mu \mathrm{g} /$ $\mathrm{ml})$, lo cual también ha sido descrito por diversos autores 28,34 . Nuestro estudio evidenció que E faecalis presenta el mayor nivel de resistencia a los aminoglicósidos, lo que no concuerda con lo descrito por González y col ${ }^{35}$. Es importante hacer una adecuada identificación de las especies de enterococos y evaluar en forma periódica la resistencia a los antibióticos aminoglicósidos. Los antibióticos aminoglicósidos se asocian con antibióticos ß-lactámicos para obtener efectos sinérgicos frente a infecciones por enterococos, pero éstos pueden usarse solamente cuando la resistencia es de bajo nivel. Ha sido descrito que la resistencia a los antibióticos aminoglicósidos parece estar mediada por la presencia de enzimas inactivantes de aminoglicósidos ${ }^{36-38}$. 
Ph-Plate ha sido usada con éxito en estudios ecológicos y epidemiológicos sobre enteroco$\cos ^{14,39}$. Los resultados de la tipificación de las cepas de enterococos, por el sistema Ph-Plate, nos mostraron la existencia de una gran diversidad de fenotipos de enterococos en aguas servidas, sugiriendo una gran biodiversidad. Sin embargo, la existencia de cepas de enterococos altamente resistentes a aminoglicósidos, con idénticos fenotipos, tanto en las plantas de tratamientos como en el colector del hospital, sugiere una vía de diseminación común de estas bacterias, pudiendo provenir del medio hospitalario o del medio ambiente. Estos fenotipos de enterococos, en especial de E faecalis, E faecium y E hirae, presentan una elevada resistencia a los antibióticos aminoglicósidos estudiados.

Dos fenotipos clonales se aislaron con mayor frecuencia, fenotipo $\mathrm{C} 1$, que incluía a 21 cepas y fenotipo C6 con 7 especies, que se encontraron presentes en las plantas de tratamiento Cascal Norte y Cascal Sur y también, en las aguas del

\section{REFERENCIAS}

1. Iversen A, Kuhn I, Frankin A, Mölby R. High prevalence of vancomycin resistant enterococci in Swedish sewage. J Clin Microbiol 2002; 68: 2838-42.

2. Mundy LM, SAHM DF, GiLmore M. Relationships between enterococcal virulence and antimicrobial resistance. Clin Microbiol Rev 2000; 13: 513-22.

3. Aarestrup FM, Agerso Y, Gerner-Smidt P, Madsen M, JeNSEN LB. Comparison of antimicrobial resistence phenotypes and resistance genes in Enterococcus faecalis and Enterococcus faecium from humans in the community, broilers, and pigs in Denmarck. Diagn Microbiol Infect Dis 2000; 37: 127-37.

4. CHow JW. Emergence of vancomycin-resistant enterococci. Emerg Infect Dis 2000; 31: 586-9.

5. Cetinkaya Y, Falk P, Mayhall CG. Vancomycin resistant enterococci. Clin Microbiol Rev 2000; 13: 686-707.

6. AARESTRUP FM. Ocurrence of glycopeptide resistance among Enterococcus faecium isolates from environmental conventional and ecological poultry farm. Microbial Drug Resistance 1995; 1: 255-7. colector del Hospital Regional de Antofagasta. Estas cepas de enterococos resistentes a los antibióticos están circulando, vehiculizadas por el agua, en la población y medio ambiente de Antofagasta. Estas cepas presentan multirresistencia, es decir, resistencia a varios antibióticos conjuntamente, lo que puede representar un riesgo potencial para pacientes hospitalizados, que pueden desarrollar infecciones intrahospitalarias por cepas multirresistentes y en las que fracasa la terapia antibiótica convencional ${ }^{33,40}$.

Estos resultados nos muestran la existencia de un fenotipo predominante de enterococos resistente a los antibióticos, que puede encontrarse presente en el intestino de la población de Antofagasta y, por consiguiente, se encuentra en las aguas servidas de la ciudad. Se hace necesario realizar un estudio epidemiológico ecológico para conocer el origen de las cepas de enterococos resistentes y la vía de diseminación, para ello deben examinarse muestras de diferentes ambientes.

7. Aarestrup FM, Hasman H, Jensen LB, Moreno $M$, Herrero IA, Domínguez L et al. Antimicrobial resistance among enterococci from pigs in three European countries. Appl Environ Microbiol 2002; 68: 4127-9.

8. Kuare I, Konstabel C, Badstubner D, Werner G, WiTte W. Ocurrence and spread of antibiotic resistances in Enterococcus faecium. Int J Food Microbiol 2003; 88: 269-90.

9. MuRRAY BE. Diversity among multidrug-resistant enterococci. Emerg Infect Dis 1998; 4: 37-47.

10. SPERA RV, FARBER BF. Multidrug-resistant Enterococcus faecium. An intreatable nosocomial pathogen. Drug 1994; 48: 678-88.

11. LINDEN PK, MILER CB. Vancomycin-resistant enterococci: the clinical effect of a common nosocomial pathogen. Diagn Microbiol Infect Dis 1999; 33: 113-20.

12. Rice LB. Emergence of vancomicin-resistant enterococci. Emerg Infect Dis 2001; 7: 183-7.

13. Rice LB, Hutton-Thomas R, Lakticova V, Helfand MS, DONSKEY CJ. B-lactam antibiotics and gastrointestinal colonization with vancomycin-resistant. J Infect Dis 2004; 189: 1113-8. 
14. Kuhn I, Iversen A, Burman LG, Olsson-Lluequist, Frankin A, FinN M et al. Epidemiology and ecology of enterococci, with special reference to antibiotic resistant strains, in animals, humans and the environment. Int J Antimicrob Agents 2000; 14: 337-42.

15. Wegener HC, Aarestrup FM, Jensen LB, Hammerum AM, BAGER F. Use of antimicrobial growth promoters in food animals and Enterococcus faecium resistance to therapeutic antimicrobial drugs in Europe. Emerg Infect Dis 1999; 5: 329-35.

16. Iversen A, Kuhn I, Rahman M, Frankun A, Burman LG, OLsSon-Liljequist B ET aL. Evidence for transmission between humans and the environment of a nosocomial strain of Enterococcus faecium. Environ Microbiol 2004; 6: 55-9.

17. Bonten MJM, Hayden MK, Nathan C, Vanvoorhis J, Matushek M, Slaughter S et al. Epidemiology of colonization of patients and environment with vancomycin-resistant enterococci. Lancet 1996; 348: 1615-9.

18. Iversen A, Kuhn I, Rahman M, Frankun A, Burman LG, Olsson-Lijequist B ET aL. Evidence for transmission between humans and the environment of a nosocomial strain of Enterococcus faecium. Environ Microbiol 2004; 6: 55-9.

19. Torres C, Reguera Ja, San Martín MJ, Pérez-Díaz JC, BAQUERO F. vanA-mediated vancomycin-resistant Enterococcus spp in sewage. J Antimicrob Chemother 1994; 33: 553-61.

20. Vilanova X, Manero A, Cerda-Cueluar M, Blanch AR. The effect of a sewage treatment plant effluent on the faecal coliforms and enterococci populations of the reception river waters. J Appl Microbiol 2002; 92: 210-4.

21. Kuhn I, Iversen A, Burman LG, Olsson-LijJequist B, Frankin A, Finn M et al. Comparison of enterococcal populations in animals, humans, and the environment-a European study. Int J Food Microbiol 2003; 88: 133-45.

22. Blanch AR, Capun JL, Iversen I, Kühn I, Manero A et AL. Comparison of enterococcal populations related to urban and hospital wastewater in various climatic and geographic European regions. J Appl Microbiol 2003; 94: 994-1002.

23. Silva J, Asserelia L, Bolados N, Leyton J. Tipificación y estudio de la resistencia a drogas antimicrobianas de cepas de Enterococcus spp aisladas en hospitales de la zona norte. XXVI Congreso Chileno de Microbiología, Valparaíso. Acta Microbiológica 2004; 10: 88.
24. Kühn I, Iversen A, Mö山by R. The Phene-Plate system for studies of the diversity of enterococcal populations from the food chain and the environment. Int J Food Microbiol 2003; 88: 189-96.

25. Manero A, Blanch A. Identification of Enterococcus spp with a biochemical key. Appl Environ Microbiol 1999; 65; 4425-30.

26. Silva J, Avelo C, Matamoro F, Vilagra L, Rojas V, SANDOVAL G. Resistencia a antimicrobianos en diferentes biotipos de Acinetobacter baumannii aislados en el norte de Chile. Rev Méd Chile 1999; 27: 926-34.

27. NCCLS. Methods for dilution antimicrobial susceptibility test for bacteria that grow aerobically. PA 2000.

28. Катоuц M, Foo E, KüHn I, Mö山by R. Evaluation of the Phene Plate generalized microplate for metabolic fingerprinting and for measuring fermentative capacity of mixed bacterial populations. J Appl Microbiol 1997; 82: 511-8.

29. Giglio MS, Pinto ME, Córdova E, Escandar P, Waman C. Identificación de especies de Enterococcus en muestras clínicas y susceptibilidad a agentes antimicrobianos. Rev Méd Chile 1996; 124: 70-6.

30. Mela S, Sepúlveda M, Acosta P, Bello H, Domínguez M, GonZÁlezZ G et AL. Aislamiento de Enterococcus faecium resistente a vancomicina con genotipo vanB Hospital Clínico Regional de Concepción. Rev Chil Infectol 2002; 19: 32-6.

31. http://www.ispch.cl

32. Lopardo H, Casimir L, Hernández C, Rubeglo EA. Isolation of three strains of beta-lactamase-producing Enterococcus faecalis in Argentina. Eur J Clin Microbiol Infect Dis 1990; 9: 402-5.

33. MurRAY BE. B-lactamase-producing enterococci. Antimicrob Ag Chemother 1992; 36: 2355-9.

34. Снош JW. Aminoglycoside resistance in enterococci. Clin Infect D 2000; 331: 586-9.

35. González M, de Miguel I, Cañas A, Martín A. Resistencia a antibióticos en aislados del género Enterococcus. Rev Esp Quimioterap 2000; 813: 412-6.

36. Chow JW, Kak V, You I, Kao SJ, Petrin J, Clewell DB ET AL. Aminoglycoside resistance genes aph(2")-Ib and aac(6')-Im detected together in strains of both Escherichia coli and Enterococcus faecium. Antimicrob Agents Chemother 2001; 45: 2691-4. 
37. Del Campo R, Tenorio C, Rubio C, Castimo J, Torres C, GómEz-Lus R. Aminoglycoside-modifying enzymes in high-level streptomycin and gentamicin resistant Enterococcus spp in Spain. Int J Antimicrob Agents 2000; 15: 221 -6.

38. Del Campo R, Ruiz-Garbajosa P, Sánchez-Moreno MP, Baquero F, Torres C, Canton R et al. Antimicrobial resistance in recent fecal enterococci from healthy volunteers and food handlers in Spain: genes and phenotypes. Microb Drug Resist 2003; 9: 47-60.
39. KüHn I, Burman LG, HaEggman S, Tuluus K, MuRRAY BE. Biochemical fingerprinting for epidemiological typing of enterococci compared with ribotyping and pulsed-field gel electrophoresis of DNA. J Clin Microbiol 1995; 33: 2812-17.

40. LiNDen PK, MiLAR CB. Vancomycin-resistant enterococci: the clinical effect of a common nosocomial pathogen. Diagn Microbiol Infect Dis 1993; 33: $113-20$.

AgRAdECIMIENTOS

Agradecemos la gentileza de la Dra. Rosa del Campo del Hospital Ramón y Cajal de Madrid, España, por la revisión del manuscrito y sus importantes comentarios. 